\title{
Vertical-Cavity Surface-Emitting Laser Light-Current Characteristic at Constant Internal Temperature
}

\author{
Andreas Hangauer, Member, IEEE, Jia Chen, Member, IEEE, and Markus-Christian Amann, Fellow, IEEE
}

\begin{abstract}
Two continuous-wave measurement methods for the laser $P-I$-characteristic at a constant internal temperature are presented (constant average cavity and constant junction (active region) temperature). The methods are used to correctly quantify the temperature dependence of threshold current and differential quantum efficiency without need for pulsed measurements. It is found that the deviations between both temperatures can be as high as $5 \mathrm{~K}$ in a 1390 -nm InP-based buried tunnel junction vertical-cavity surface-emitting laser (BTJ-VCSEL), which is the reason for the slight sublinearity of the characteristics at constant cavity temperature.
\end{abstract}

Index Terms - Thermal variables measurement, threshold current, thermal resistance, vertical-cavity surface-emitting lasers.

\section{INTRODUCTION}

$\mathbf{V}$ ERTICAL-CAVITY surface-emitting lasers (VCSELs) have intrinsic advantages like low power consumption, longitudinal single-mode operation and low-cost potential over conventional edge-emitting distributed feedback (DFB) lasers. However, VCSELs have generally lower output power and exhibit a stronger effect of self-heating. This means that the junction temperature in a VCSEL is higher than in a DFB laser at typical operating currents and equal heat sink temperature. Since both the differential quantum efficiency ("slope" of the $P-I$-characteristic) and the threshold current are junction temperature $T_{j c n}$ dependent, self-heating causes the bending observed in the $P-I$-characteristic of VCSELs including the thermal rollover.

It is expected that the dependence of VCSEL output power $P$ on current $I$ at constant internal temperature has much less curvature than at the ordinary $P-I$ characteristic at constant heatsink temperature $T_{S}$, i.e. the output power is almost linear to injection current without self-heating. The advantage of knowing the junction temperature $T_{j c n}$ is that a general model for the output power of semiconductor lasers can be stated [1]:

$$
P\left(I, T_{j c n}\right)=S\left(T_{j c n}\right) \cdot\left(I-I_{t h}\left(T_{j c n}\right)\right)
$$

Manuscript received March 16, 2011; revised May 31, 2011; accepted June 17, 2011. Date of publication June 23, 2011; date of current version August 24, 2011.

A. Hangauer is with the Walter Schottky Institute, Technical University of Munich, Garching 85748, Germany, and also with Corporate Research and Technologies, Siemens AG, Munich 80200, Germany (e-mail: andreas.hangauer@wsi.tum.de).

J. Chen and M.-C. Amann are with the Walter Schottky Institute, Technical University of Munich, Garching 85748, Germany (e-mail: jia.chen@wsi.tum.de; mcamann@wsi.tum.de).

Color versions of one or more of the figures in this letter are available online at http://ieeexplore.ieee.org.

Digital Object Identifier 10.1109/LPT.2011.2160389 with $I_{t h}\left(T_{j c n}\right)$ the laser threshold current and $S\left(T_{j c n}\right)$ the corresponding slope that to a first approximation only depend on the active region temperature $T_{j c n}$. The slope $S$ is proportional to the differential quantum efficiency $\eta_{e}$ of the laser $\left(\eta_{e}=\right.$ $S e /(h \nu)$, with $\nu$ the emission frequency, $h$ the Planck constant and $e$ the elementary charge).

In literature internal temperature is determined by comparing continuous wave laser characteristics (output power $P$, laser voltage $U$ or emission wavelength $\lambda$ ) with pulsed measurements [2] or from an estimation of laser thermal resistance [3]. A pulsed measurement requires a broadband measurement setup which is not available for all wavelengths and also has other disadvantages. The laser thermal resistance is an effective value and typically changes with bias current $I$ and internal temperature due to variations of the heat source distribution and thermal material properties. For precise determination of the internal temperature this variation has to be taken into account. Furthermore, there is a certain inaccuracy in the term "internal temperature" because the active region temperature $T_{j c n}$ may be different from the average cavity temperature $T_{\text {cav }}$ in a VCSEL structure. The lack of literature on this issues was part of the motivation for the development of a continuous-wave (non-pulsed) method for characterization of the $P-I$-characteristic at constant junction temperature which is presented in this letter. In summary we will present two continuous wave methods that do not have these disadvantages mentioned above and differentiate between cavity temperature (method 1 ) and junction temperature (method 2).

\section{Measurement Methods}

In this section the two continuous wave measurement methods and measurement results for $P-I$-characteristics at constant junction $P\left(I, T_{j c n}\right)$ and cavity temperature $P\left(I, T_{\text {cav }}\right)$ are presented and demonstrated on a standard InP-based buried tunnel junction (BTJ) VCSEL [4] emitting at $1390 \mathrm{~nm}$. In principle the ordinary measurement data $P\left(I, T_{S}\right)$ (output power $P$ at current $I$ and heat-sink temperature $\left.T_{S}\right)$ is used to extract this information. To compensate for self-heating, the heat-sink temperature $T_{S}$ has to decrease if the injection current $I$ is increased.

For finding the $P-I$-characteristic at constant $T_{j c n}$ or $T_{\text {cav }}$, trajectories in the $\left(I, T_{S}\right)$ plane have to be found, where the corresponding internal temperature is constant. For a graphical illustration of this please refer to the obtained results in Fig. 2 and Fig. 3. The absolute temperature value is determined by extrapolation of the trajectories to $I=0$ (because there $T_{j c n}=$ $T_{\text {cav }}=T_{S}$ ). Although not needed here, the effective differential laser thermal resistance $R_{t h}$ can be determined by the tangent 


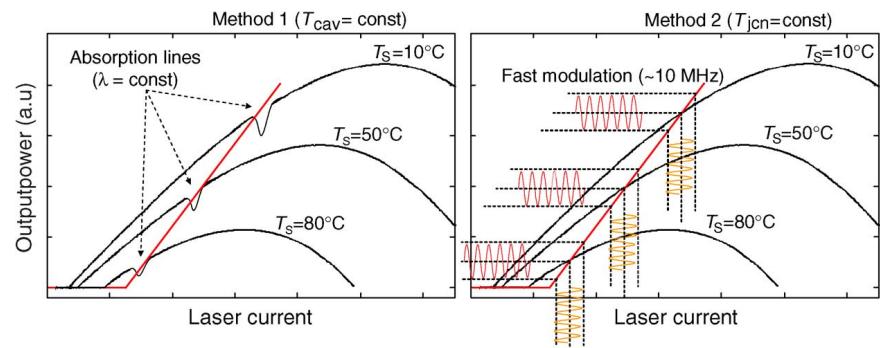

Fig. 1. Schematical explanation of method 1), where $\lambda=$ const, known from gas absorption lines corresponds to $T_{\text {cav }}=$ const and method 2), where a high-speed modulation is used to get the slope $S$ of the $P-I$-characteristic at $T_{j c n}=$ const. The reconstruction of the $P-I$ characteristic at constant internal temperature is shown in red.

of the trajectory $d T_{S} / d I$ and the dissipated power in the device $P_{\text {diss }}=U I-P(I)$, by $R_{t h}=\left(d T_{S} / d I\right) /\left(d P_{\text {diss }} / d I\right)$.

\section{A. Method 1: $T_{\text {cav }}=$ const}

The emission wavelength is dominantly determined by the intrinsic thermal tuning, i.e. the average cavity temperature [5]. For an illustration see Fig. 1 (left). Trajectories

$$
\left(I, T_{S}\right) \quad \text { where } \quad \lambda\left(I, T_{S}\right)=\text { const }
$$

are thus trajectories of constant cavity temperature (see e.g. Fig. 2 in the results section). Here $\lambda\left(I, T_{S}\right)$ was determined by identifying the absorption lines in the measured $P-I$-characteristics and comparing the positions with known wavelengths from a spectral database [6].

\section{B. Method 2: $T_{j c n}=$ const}

By applying a small-signal modulation with frequency well above the thermal cutoff frequency and below the relaxation frequency (here $f=10 \mathrm{MHz}$ [7]) the slope of the $P-I$-characteristic at constant junction temperature $\Delta P / \Delta I=S\left(I, T_{S}\right)$ is determined. Knowing the slope $S\left(I, T_{S}\right)$ and the laser output power $P\left(I, T_{S}\right)$, the full internal $P-I$-characteristic can be reconstructed. For an illustration see Fig. 1 (right). The relation $\Delta P /\left.\Delta I\right|_{f=10 \mathrm{MHz}}=S$ is easily proved, when (1) is differentiated by $I$ and the $d T_{j c n} / d I$ terms are set to zero (i.e. there is no self-heating by the fast small-signal modulation). Mathematically, the trajectory of constant junction temperature is found by iteratively solving

$$
P\left(I+\Delta I, T_{S}+\Delta T_{S}\right)=S\left(I, T_{S}\right) \Delta I+P\left(I, T_{S}\right) .
$$

for $\Delta T_{S}$ with an arbitrary but fixed current step $\Delta I<0$. There $\Delta T_{S}$ is the value the heat-sink temperature needs to be increased by when the injection current rises by $\Delta I$ to maintain constant junction temperature. The complete trajectory is found by repeatedly solving (3): First a starting point $\left(I, T_{S}\right)$ with the maximum desired current $I$ and $\Delta I<0$ is selected and second (3) numerically solved for $\Delta T_{S}$. The procedure is repeated with the new starting point $\left(I+\Delta I, T_{S}+\Delta T_{S}\right)$ until the point wanders below threshold. See Fig. 3 in the results section for the determined trajectories and the "lasing domain" surrounded by thick black lines labeled "threshold" and "laser turn-OFF".

The experimental data was determined with a gain/phase analyzer operated at $10 \mathrm{MHz}$. The cathode of the laser is connected

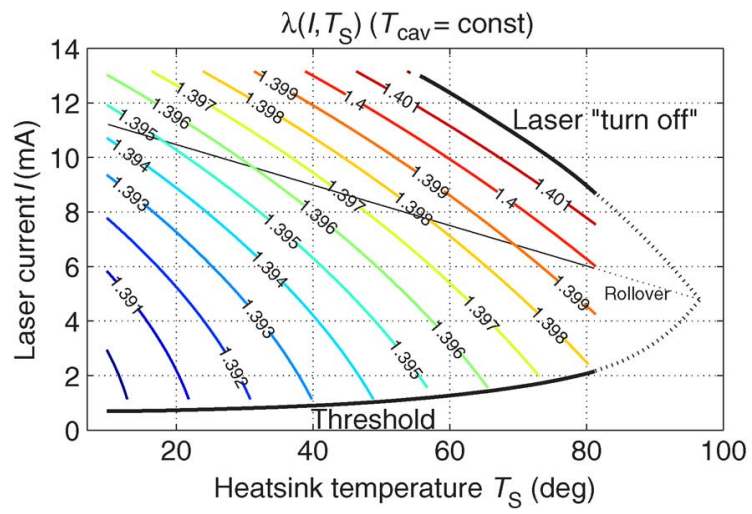

Fig. 2. Trajectories of constant cavity temperature $T_{c a v}$ (i.e., constant laser emission wavelength $\left.\lambda\left(I, T_{S}\right)\right)$ in the $\left(I, T_{S}\right)$ plane obtained with method 1 . In contrast to laser rollover, the turn-OFF happens at constant internal temperature. Due to the smoothness of the curves extrapolation to $I=0$ for absolute temperature determination is feasible.

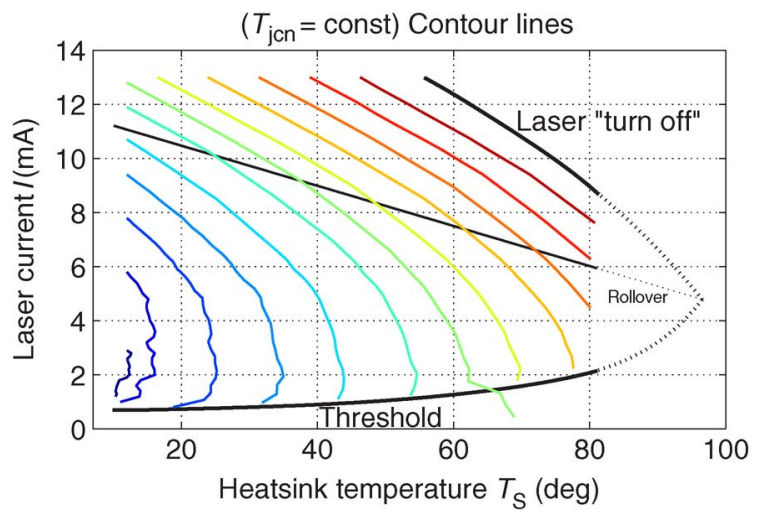

Fig. 3. Trajectories of constant junction temperature $T_{j c n}$, reconstructed from the measured slope $S\left(I . T_{S}\right)$ and emission power data $P\left(I, T_{S}\right)$, using method 2 [iteratively solving (3)]. If $S$ is close to $d P / d I$, the method becomes inexact, which makes extrapolation to $I=0$ for absolute temperature determination more difficult.

to the $50 \Omega$ terminated reference input (for measuring $\Delta I$ ) and the photodiode is connected to the $50 \Omega$ terminated test input (for measuring $\Delta P$ ). This configuration directly measures the slope $S\left(I, T_{S}\right)=\Delta P / \Delta I$, while simultaneously the $P\left(I, T_{S}\right)$ curves are determined as the DC components of the reference and test signals.

\section{EXPERIMENTAL RESULTS}

The determined trajectories using both methods are shown in Fig. 2 and Fig. 3. The resulting $P-I$-characteristics are shown in Fig. 4 and Fig. 5, respectively. Although the curves in Fig. 4 and Fig. 5 remain a little bent (in Fig. 4 more than in Fig. 5), the output power is far more linear to injection current than for the ordinary $P-I$-characteristic suffering from self-heating (dashed lines). This clearly proves that self-heating is the dominant cause of the strong nonlinearity of the VCSEL $P-I$-characteristic. The slope and threshold of the $P-I$-characteristic is plotted against temperature in Fig. 6. Recalling (1), it is apparent that the rise in threshold only contributes partly to the nonlinearity of the ordinary $P-I$-characteristic, because also the slope $S$ is strongly decreasing with internal temperature. This very nicely explains the fact that the laser turns OFF at a specific internal temperature: The turn-OFF happens at approximately the 


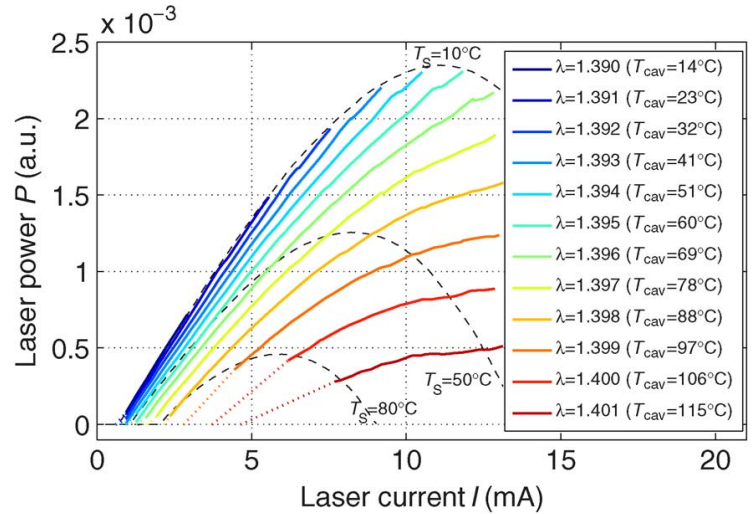

Fig. 4. Laser power at constant cavity temperature $T_{c a v}$ (Method 1), corresponding to the trajectories in Fig. 2. For comparison some ordinary $P-I$-characteristics are also shown (dashed, black).

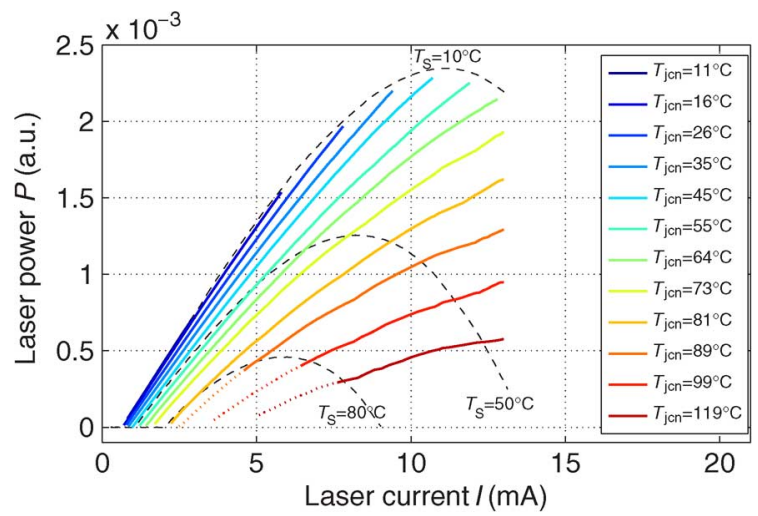

Fig. 5. Laser power at constant junction temperature $T_{j c n}$ (Method 2), corresponding to the trajectories in Fig. 3. For comparison some ordinary $P-I$-characteristics are also shown (dashed, black).

temperature where the slope reaches zero and according to (1) no light is generated. The actual value of $130^{\circ} \mathrm{C}$ is obtained by extrapolation in Fig. 6. This effect is also found in GaAs-based VCSELs [8] and in edge-emitting lasers where the differential quantum efficiency ("slope") reaches zero at a certain temperature. This is either due to lower internal efficency, i.e. less current is flowing through the active region or increased absorption losses. For the latter free-carrier absorption or intervalence band absorption are typically dominant contributions when the carrier density rises with temperature. Note, that if laser turn-OFF was caused by the threshold current $I_{t h}\left(T_{j c n}\right)$ to reach $I$ (see (1)) the laser-turn OFF would not happen at a constant internal temperature. Such a behavior is for instance observed for the laser rollover (see Fig. 2 and Fig. 3) which happens at different internal temperatures.

Fitting the empirical model

$$
S\left(T_{c a v / j c n}\right)=S(0)\left(1+\alpha T_{c a v / j c n}\right)
$$

we obtain $\alpha=-0.0074 \mathrm{~K}^{-1}$ (Method 1) and $\alpha=$ $-0.0070 \mathrm{~K}^{-1}$ (Method 2) with deviation less than $12 \%$. The deviation is attributed to the fact that generally $T_{c a v} \neq T_{j c n}$. This is evident from the higher curvature of the characteristics with $T_{c a v}=$ const. For currents around $I_{t h}$ or around $12 \mathrm{~mA}$ we

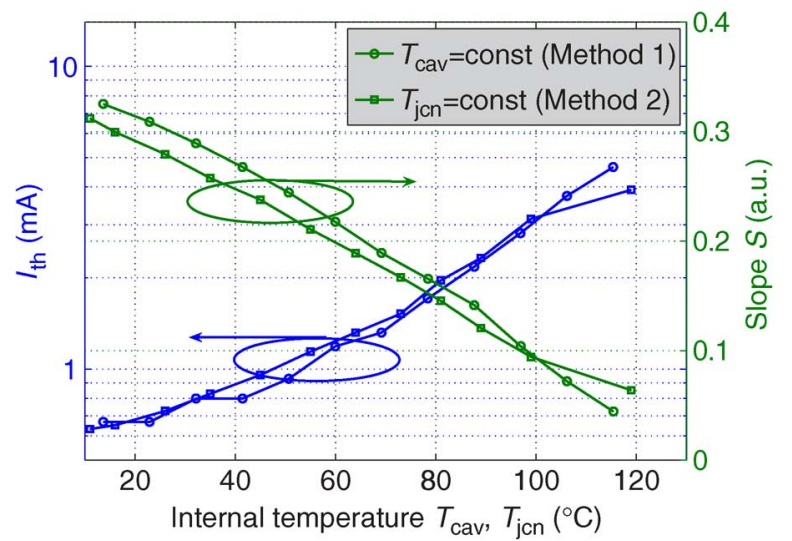

Fig. 6. Threshold current $I_{t h}$ and the (average) slope $S$ of the curves in Fig. 4 versus internal temperature $T_{j c n}$ or $T_{c a v}$. Extrapolating to about $130^{\circ} \mathrm{C}$ the laser turns OFF (slope becomes zero).

have $T_{\text {cav }} \approx T_{j c n}$, however around 5-7 mA $T_{j c n} \approx T_{c a v}+5 \mathrm{~K}$ can be estimated when superimposing Fig. 4 and Fig. 5.

\section{CONCLUSION}

Concluding, the presented two measurement methods allow for determination of the PI characteristics at constant cavity or junction temperature without a pulsed measurement. The temperature dependence of the laser threshold current and differential quantum efficiency can be correctly quantified without self-heating. In an InP-based VCSEL both temperatures differ by up to $5^{\circ}$ which causes the residual bending of the $T_{\text {cav }}=$ const characteristics. Furthermore, laser turn-OFF happens in contrast to rollover at constant internal temperature.

\section{ACKNOWLEDGMENT}

The authors would like to thank S. Arafin for valuable comments.

\section{REFERENCES}

[1] L. A. Coldren and S. W. Corzine, Diode Lasers and Photonic Integrated Circuits. Hoboken, NJ: Wiley, 1995.

[2] T. Paoli, "A new technique for measuring the thermal impedance of junction lasers," IEEE J. Quantum Electron., vol. 11, no. 7, pp. 498-503, Jul. 1975.

[3] H. Moench, J. Baier, S. Gronenborn, J. Kolb, M. Miller, P. Pekarski, M. Schemmann, and A. Valster, "Advanced characterization techniques for high power VCSELs," Proc. SPIE, vol. 7615, no. 76150G, 2010.

[4] M.-C. Amann and M. Ortsiefer, "Long-wavelength $(\lambda \geq 1.3 \mu \mathrm{m})$ InGaAlAs-InP vertical-cavity surface-emitting lasers for applications in optical communication and sensing," Phys. Status Solidi A, vol. 203, pp. 3538-3544, Aug. 2006.

[5] A. Hangauer, J. Chen, R. Strzoda, and M.-C. Amann, "The frequency modulation response of vertical-cavity surface-emitting lasers: Experiment and theory," IEEE J. Sel. Topics Quantum Electron., 2011, DOI: 10.1109/JSTQE.2011.2110640, to be published.

[6] L. Rothman et al., "The HITRAN 2004 molecular spectroscopic database," J. Quantitative Spectroscopy and Radiative Transfer, vol. 96, no. 2, pp. 139-204, Dec. 2005.

[7] J. Chen, A. Hangauer, R. Strzoda, and M.-C. Amann, "Experimental characterization of the frequency modulation behavior of vertical cavity surface emitting lasers," Appl. Phys. Lett., vol. 91, no. 14, p. 141105 , Oct. 2007.

[8] B. Weigl, M. Grabherr, C. Jung, R. Jager, G. Reiner, R. Michalzik, D. Sowada, and K. Ebeling, "High-performance oxide-confined GaAs VCSELs," IEEE J. Sel. Topics Quantum Electron., vol. 3, no. 2, pp. 409-415, Apr. 1997. 